\title{
Multiple sclerosis presented as clinically isolated syndrome: the need for early diagnosis and treatment
}

\author{
Sigliti-Henrietta Pelidou \\ Sotirios Giannopoulos \\ Sotiria Tzavidi \\ Georgios Lagos \\ Athanassios P Kyritsis
}

Department of Neurology, University of Ioannina School of Medicine, Greece
Correspondence: Sotirios Giannopoulos Dept., of Neurology, University of loannina School of Medicine, University Campus loannina, 45 I I0, Greece

Tel +302651097514

$\mathrm{Fax}+302651097011$

Emailsgiannop@uoi.gr
Objective: To aid in the timely diagnosis of patients who present with clinically isolated syndrome (CIS).

Patients and methods: We studied 25 patients (18 women, 7 men), originally presented in our clinic with a CIS suggestive of multiple sclerosis (MS). All patients underwent the full investigation procedure including routine tests, serology, cerebrospinal fluid (CSF) examinations, evoked potentials (EPs), and magnetic resonance imaging (MRI) of brain and cervical spinal cord. Patients were imaged at baseline, and every three months thereafter up to a year.

Results: The CIS was consisted of optic neuritis in 12 cases, incomplete transverse myelitis (ITM) in 7 cases, Lhermitte sign in 2 cases, internuclear ophthalmoplegia (INO) in 2 cases, mild brainstem syndrome in 1 case, and tonic-clonic seizures in 1 case. Using the baseline and three-month scans 18/25 (72\%) patients developed definite MS in one year of follow up while $7(28 \%)$ had no further findings during this observation period. Immunomodulatory treatments were applied to all definite MS patients.

Conclusion: In light of new treatments available, MRIs at 3 month intervals are helpful to obtain the definite diagnosis of MS as early as possible.

Keywords: multiple sclerosis, clinically isolated syndrome, optic neuritis, transverse myelitis

\section{Introduction}

Multiple sclerosis (MS) is a clinical diagnosis based on the dissemination of lesions of the central nervous system (CNS) in time and space (Poser and Brinar 2001). Recent proposed diagnostic criteria for MS utilize MRI findings to document dissemination of CNS lesions in time and space (McDonald et al 2001; Polman et al 2005). Optic neuritis (ON) may be the heralding manifestation of MS (ONSG 1991; Hickman et al 2002). More than $50 \%$ of adult patients who present with isolated ON will eventually develop other signs of MS (ONSG 1997). The risk stratification for the future development of MS in patients presenting with ON can be assessed by the number of white matter lesions on the baseline cerebral MRI study (Bhatti et al 2005). The risk is increased in women and in those patients who have oligoclonal bands (OCB) in the cerebrospinal fluid (CSF) (Ghezzi et al 1999).

Incomplete transverse myelitis (ITM) can be the presenting feature of MS. Patients with ITM who develop MS are more likely to have asymmetric clinical findings, predominant sensory symptoms with relative sparing of motor systems, spinal cord lesions extending over less than two spinal segments, abnormal brain MRI, and OCB in the CSF (Miller et al 1989; TMC 2002). Measurement of spinal cord atrophy that reflects destructive, irreversible pathology in patients presenting with ITM has important implications for the early treatment of MS (Lin et al 2004). 
Central nervous system involvement in MS may be difficult to differentiate from other autoimmune diseases such as systemic lupus erythematosus (SLE), antiphospholipid syndrome (APS), and Sjogren syndrome (SS). Thus, a large number of patients with APS or SLE may be misdiagnosed as MS, but the exact proportion is unknown and may receive inappropriate treatment (Ferreira et al 2005). A retrospective study in 82 patients with primary Sjogren syndrome reported that $40.2 \%$ had brain involvement, $15.8 \%$ optic neuropathy, and $35.4 \%$ spinal cord involvement (Delalande 2004). Thus, the differential diagnosis may require careful assessment of all clinical and MRI findings, serological results, and CSF analysis (Reske et al 2005).

Our objective was to aid in the timely diagnosis of MS in patients who presented with CIS and thus employ the proper treatment early during the course of the disease.

\section{Patients and methods}

We studied 25 consecutive patients ( 18 women, 7 men), age 30.6 (range: 17-49) presented in our clinic with CIS suggestive of MS. The inclusion and exclusion criteria of the CIS patients are listed in Table 1. The clinical, laboratory, and imaging findings were studied in all patients. Laboratory investigations included complete blood count, serum values of liver enzymes, bilirubin, albumin, glucose, creatinine, and urine analysis. In addition, erythrocyte sedimentation rate, C-reactive protein, antinuclear antibodies (ANA), double-stranded DNA (ds-DNA), complement $\mathrm{C} 3 / \mathrm{C} 4$, and anti-cardiolipin (ACL) antibodies were also perfomed.

All patients were evaluated for MS with CSF serology (cells, protein, glucose, IgG index), visual evoked potentials (VEPs), somatosensory evoked potentials (SEPs), and brain and cervical spinal cord MRI. Patients were imaged

Table I List of inclusion and exclusion criteria

\begin{tabular}{ll}
\hline Inclusion criteria & Exclusion criteria \\
\hline Optic neuritis & Head trauma \\
Diplopia & Neuropsychiatric disorder \\
Internuclear opthalmoplegia & Diabetes mellitus \\
Myelitis & Hypertension \\
Hemiparesis-paraparesis & Cardiac failure \\
Dysarthria & Hepatic failure \\
Sensory symptoms & Renal failure \\
MRI criteria: & Thalassemia, \\
a. I GD enhancing lesion & Sickle cell disease, \\
b. I infratentorial lesion & Iron deficiency, \\
c. I juxtacortical lesion & BI2 deficiency \\
d. 3 periventricular lesions & \\
\hline
\end{tabular}

Abbreviation: MRI, magnetic resonance imaging. at baseline, at three month intervals, and one year. CSF oligoclonal bands were not included in the evaluation since it is not an available test in our hospital laboratory. For MS diagnosis, the revised McDonald criteria were employed (Polman et al 2005). Dissemination in space was demonstrated by either MRI alone when three of the following conditions were met: a) at least one gadolinium-enhancing lesion or nine T2 hyperintense lesions if there was no gadolinium enhancing lesion; b) at least one infratentorial lesion; c) at least one juxtacortical lesion; and d) at least three periventricular lesions, or two or more MRI-detected lesions consistent with MS plus elevated CSF IgG index. Dissemination in time was demonstrated by either MRI (detection of gadolinium enhancing abnormality at least 3 months after the onset of the initial clinical event and if not at the site corresponding to the initial event detection of a new T2 lesion if it appeared at any time compared with the baseline scan performed at least 30 days after the onset of the initial clinical event), or by a second clinical attack.

None of the studied patients had history of head trauma, neuropsychiatric disorder, diabetes mellitus, hypertension, thalassemia, iron and B12 deficiency, cardiac, hepatic and renal failure (Table 1). Finally, the protocol was approved by the Institutional Review Board Committee.

\section{Results}

The characteristics of all CIS patients are depicted in Table 2 . There were 25 patients (18 women, 7 men), of 30.6 years mean age (range 17-49). The mean age of patients at onset of CIS varied from 26.9 years for ON to 29.2 years for the patient with seizures. The mean follow-up time was 18.9 months in patients with ON, 17.5 months in patients with ITM, 18 months in patients with brainstem syndrome. The mean disease severity on the expanded disability status scale (EDSS) performed at least one month after the CIS varied from 0.41 to 1 . Only 12 woman had isolated ON, 7 had ITM, 3 had mild brainstem syndrome (2 with internuclear ophthalmoplegia (INO), 1 with dysarthria), and 1 had sudden onset of tonic-clonic seizures. The predominant neurological examination findings during the acute phase of the CIS were: reduced unilateral visual acuity in the patients with $\mathrm{ON}$, paraparesis, Lhermitte sign, sensory symptoms, diplopia, dysarthria, and hemiparesis as shown in Table 3.

The paraclinical data of the patients are shown in Table 4. Twenty four patients had abnormal brain MRI consisted of periventricular MS-like white matter lesions and 9 (36\%) patients had evidence of myelitis. Both brain and cervical cord MRI were positive in 7 cases $(28 \%)$. 
Table 2 Characteristics of CIS patients

\begin{tabular}{llll}
\hline & ON & ITM & Brainstem syndrome \\
\hline Sex ratio (female/male) & $12 / 0$ & $3 / 4$ & $2 / 1$ \\
Age at diagnosis mean +I- SD (range) & $29.5 \pm 16(17-46)$ & $28.9 \pm 8.7(20-42)$ & $29.6 \pm 12(24-49)$ \\
Age at onset of CIS mean +I- SD (range) & $26.9 \pm 10.3(17-43)$ & $28.7 \pm 9.1(19-37)$ & $29.1 \pm 9.6(21-48)$ \\
Follow up time mean months (range) & $18.9(9-60)$ & $17.5(3-26)$ & $18(I 7-29)$ \\
Duration of disease mean months, (range) & $20.7(9-61)$ & $19.3(4-26)$ & $19.5(17-29)$ \\
Disease severity on EDSS mean (range) & $0.4 I(0-1)$ & $0.6(0-2.5)$ & $1(1-1)$ \\
\hline
\end{tabular}

Abbreviations: CIS, clinically isolated syndrome; ON, optic neuritis; ITM, incomplete transverse myelitis; EDSS, expanded disability status scale.

Note: ${ }^{a}$ EDSS was assessed at least one month after the CIS.

VEP's were abnormal in $16(64 \%)$ and SEP's in $6(24 \%)$ patients. Nineteen patients $(76 \%)$ had increased $\operatorname{IgG}$ index, while in 6 patients it was normal.

Serological features of the patients are demonstrated in Table 5. ANA were positive in $5(20 \%)$ and anti-ds-DNA in $2(8 \%)$ of patients, but no patient had low complement (C3 or C4). ACL-ab was positive in only $3(12 \%)$ patients.

Using the baseline and three-month MRI scans and the clinical and paraclinical data, $72 \%$ of patients with CIS developed definite MS according to the revised McDonald's criteria within one year, while $28 \%$ had no further neurological and MRI findings during this observation period.

\section{Discussion}

Patients with CIS may develop MS (Miller et al 2005). Clinically the identification of patients with a CIS at high risk to develop clinically definite MS remains difficult (Kieseier et al 2005). Monthly brain MRI scans in patients with CIS showed that the majority of patients with an abnormal baseline scan were diagnosed with MS after three months (Pestalozza et al 2005). Application of the new McDonald criteria to patients with CIS suggestive of MS doubled the rate of diagnosis of MS within one year of presentation (Dalton et al 2002). It has been suggested that monthly triple-dose Gd-enhanced

Table 3 Clinical data of CIS patients

\begin{tabular}{ll}
\hline Neurological symptoms and signs & No. of patients (\%) \\
\hline Optic neuritis & $12(48 \%)$ \\
Myelitis & $7(28 \%)$ \\
Internuclear opthalmoplegia & $2(8 \%)$ \\
Seizures & $1(4 \%)$ \\
Paraparesis & $5(25 \%)$ \\
Lhermitte sign & $2(8 \%)$ \\
Sensory symptoms & $9(36 \%)$ \\
Diplopia & $3(12 \%)$ \\
Dysarthria & $1(4 \%)$ \\
Hemiparesis & $1(4 \%)$ \\
\hline
\end{tabular}

Abbreviations: CIS, clinically isolated syndrome.
MRIs accurately detected inflammation which is an important factor in the pathogenesis of brain tissue loss in CIS patients (Paolillo et al 2004).

Although a CIS could be indicative of MS, it might occasionally be the first manifestation of systemic autoimmune diseases such as SLE, APS, and SS. In these cases not only the clinical presentation but the MRI findings may be similar (Theodoridou and Settas 2006). Thus, serology tests, eg, ANA, C3, C4, and antiphospholipid antibodies, could help in the differential diagnosis of MS from the various connective tissue diseases. In the present study $20 \%$ of CIS patients had positive ANA and a few patients had other autoantibodies but there were no other findings to indicate evidence of SLE, primary SS, and APS according to the currently used criteria (Tan et al 1982; Wilson et al 1999; Vital et al 2002). Presence of various autoantibodies such as ANA in the serum of patients with MS is a frequent finding (Specaile et al 2000). Brain MRI can be inconclusive in patients with CIS but intrathecal synthesis of OCB are helpful in establishing the diagnosis of MS (Sastre-Garriga et al 2003; Rot and Mesec 2006), thus the lack of OCB measurements is a limitation of our study. A new OCB test may also improve the conversion of a CIS to MS (Masjuan et al 2006). Prediction of conversion from CIS to MS can be improved if CSF
Table 4 Paraclinical data of CIS patients: Initial 2/25

\begin{tabular}{lll}
\hline Parameters & $(+)$ & $(-)$ \\
\hline Initial brain MRI & $23 / 25(92 \%)$ & $2 / 25(8 \%)$ \\
Brain MRI $^{a}$ & $24 / 25(96 \%)$ & $1 / 25(4 \%)$ \\
Cervical SC MRI $^{a}$ & $9(36 \%)$ & $16(64 \%)$ \\
VEP's & $16 / 25(64 \%)$ & $9 / 25(36 \%)$ \\
SEP's & $6 / 25(24 \%)$ & $19 / 25(76 \%)$ \\
CSFb/WBC $^{\text {CSFb/IgG INDEX }}$ & $1 / 25(4 \%)$ & $24 / 25(96 \%)$ \\
\hline
\end{tabular}

Abbreviations: CIS, clinically isolated syndrome; SC, spinal cord;VEP, visual evoked potentials; SEP, somatosensory evoked potentials; CSF, cerebrospinal fluid.

Notes: 'Brain and cervical SC MRIs were considered $(+)$ when findings were observed at any time during the 3 month intervals up to a year; ${ }^{\mathrm{b}} \mathrm{CSF}$ serology was performed during the baseline evaluation. 
Table 5 Serological features of the patients

\begin{tabular}{llll}
\hline Parameters & $(+)$ & $(-)$ & Not done \\
\hline ANA & $5(20 \%)$ & $17(68 \%)$ & $3(12 \%)$ \\
Anti-ds-DNA & $2(8 \%)$ & $17(68 \%)$ & $6(24 \%)$ \\
ACL-ab & $3(12 \%)$ & $22(88 \%)$ & 0 \\
C3/C4 & 0 & $25(100 \%)$ & 0 \\
\hline
\end{tabular}

Abbreviations: CIS, clinically isolated syndrome; ANA, antinuclear antibodies; dsDNA, double-stranded DNA;ACL-ab, anticardiolipin antibodies; C3/C4, complement fractions $\mathrm{C} 3$ and $\mathrm{C} 4$.

markers (either elevated tau or neurofilaments [NfHSMI35] are combined with MRI findings (Brettschneider at al 2006). On the contrary, serum antimyelin antibodies did not seem to permit earlier diagnosis of MS (Lim et al 2005). Intrathecal B-cell clonal expansion in the CSF of patients with CIS, often precedes both OCB and multiple MRI lesions and is associated with a high rate of conversion to definite MS (Qin et al 2003).

\section{Conclusion}

Patients with MS deserve an accurate diagnosis, because new effective immunomodulatory treatments could modify the disease progression. It is obvious that definite diagnosis of MS must be obtained as early as possible in order to initiate early immunomodulatory treatment. We believe that frequent MRI scans during the first year after the CIS facilitate the early and accurate diagnosis of definite MS.

\section{References}

Bhatti MT, Schmitt NJ, Beatty RL. 2005. Acute inflammatory demyelinating optic neuritis: current concepts in diagnosis and management. Optometry, 76:526-35.

Brettschneider J, Petzold A, Junker A, et al. 2006. Axonal damage markers in the cerebrospinal fluid of patients with clinically isolated syndrome improve predicting conversion to definite multiple sclerosis. Mult Scler, 12:143-8.

Dalton CM, Brex PA, Miszkiel KA, et al. 2002. Application of the new McDonald criteria to patients with clinically isolated syndromes suggestive of multiple sclerosis. Ann Neurol, 52:47-53.

Delalande S, deSeze S, Fauchais AL, et al. 2004. Neurologic manifestations in primary Sjogren syndrome: a study of 82 patients. Medicine, 83:280-91.

Ferreira S, D'Cruz DP, Hughes GR. 2005. Multiple sclerosis, neuropsychiatric lupus and antiphospholipid syndrome: where do we stand? Rheumatology, 44:434-42.

Ghezzi A, Martinelli V, Torri V, et al. 1999. Long-term follow-up of isolated optic neuritis: the risk of developing multiple sclerosis, its outcome, and the prognostic role of paraclinical tests. J Neurol, 246:770-75.

Hickman SJ, Dalton CM, Miller DH, et al. 2002. Management of acute optic neuritis. Lancet, 360:1953-62.

Kieseier BC, Hemmer B, Hartung HP. 2005. Multiple sclerosis - novel insights and new therapeutic strategies. Curr Opin Neurol, 18:211-20.

Lim ET, Berger T, Reindl M, et al. 2005. Anti-myelin antibodies do not allow earlier diagnosis of multiple sclerosis. Mult Scler, 11:492-4.

Lin X, Tench CR, Evangelou N, et al. 2004. Measurement of spinal cord atrophy in multiple sclerosis. J Neuroimaging, 14(Suppl 3):20S-26S.
Masjuan J, Alvarez-Cermeno JC, Garcia-Barragan N, et al. 2006. Clinically isolated syndromes: a new oligoclonal band test accurately predicts conversion to MS. Neurology, 66:576-8.

McDonald WI, Compston A, Edan G, et al. 2001. Recommended diagnostic criteria for multiple sclerosis: guidelines from the International Panel on the diagnosis of multiple sclerosis. Ann Neurol, 50:121-7.

Miller D, Barkhof F, Montalban X, et al. 2005. Clinically isolated syndromes suggestive of multiple sclerosis, part 2: non-conventional MRI, recovery processes, and management. Lancet Neurol, 4:341-8.

Miller DH, Ormerod IE, Rudge P, et al. 1989. The early risk of multiple sclerosis following isolated acute syndromes of the brain stem and spinal cord. Ann Neurol, 26:635-9.

[ONSG] Optic Neuritis Study Group. 1991. The clinical profile of optic neuritis: experience of the optic neuritis treatment trial. Arch Ophthalmol, 109:1673-78.

[ONSG] Optic Neuritis Study Group. 1997. The 5-year risk of MS after optic neuritis: experience of the optic neuritis treatment trial. Neurology, 49:1404-13.

Paolillo A, Piattella MC, Pantano P, et al. 2004. The relationship between inflammation and atrophy in clinically isolated syndromes suggestive of multiple sclerosis: a monthly MRI study after triple-dose gadoliniumDTPA. J Neurol, 251:432-9.

Pestalozza IF, Pozzilli C, Di Legge S, et al. 2005. Monthly brain magnetic resonance imaging scans in patients with clinically isolated syndrome. Mult Scler, 11:390-4.

Polman CH, Reingold SC, Edan G, et al. 2005. Diagnostic criteria for multiple sclerosis: 2005 revisions to the "McDonald Criteria". Ann Neurol, 58:840-6.

Poser CM, Brinar VV. 2001. Diagnostic criteria for multiple sclerosis. Clin Neurol Neurosurg, 103:1-11.

Qin Y, Duquette P, Zhang Y, et al. 2003. Intrathecal B-cell clonal expansion, an early sign of humoral immunity, in the cerebrospinal fluid of patients with clinically isolated syndrome suggestive of multiple sclerosis. Lab Invest, 83:1081-8.

Reske D, Petereit HF, Heiss WD. 2005. Difficulties in the differentiation of chronic inflammatory diseases of the central nervous system - value of cerebrospinal fluid analysis and immunological abnormalities in the diagnosis. Acta Neurol Scand, 112:207-13.

Rot U, Mesec A. 2006. Clinical, MRI, CSF and electrophysiological findings in different stages of multiple sclerosis. Clin Neurol Neurosurg, 108:271-4

Sastre-Garriga J, Tintore M, Rovira A, et al. 2003. Conversion to multiple sclerosis after a clinically isolated syndrome of the brainstem: cranial magnetic resonance imaging, cerebrospinal fluid and neurophysiological findings. Mult Scler, 9:39-43.

Speciale L, Saresella M, Caputo D, et al. 2000. Serum auto antibodies presence in multiple sclerosis patients treated with beta-interferon 1a and 1b. J Neurovirol, 6(Supp1 2):S57-61.

Tan EM, Cohen AS, Fries JF, et al. 1982. The 1982 revised criteria for the classification of systemic lupus erythematosus. Arthritis Rheum, 25:1271-7.

Theodoridou A, Settas L. 2006. Demyelination in rheumatic diseases. J Neurol Neurosurg Psychiatry, 77:290-5.

[TMC] Transverse Myelitis Consortium Working Group. 2002. Proposed diagnostic criteria and nosology of acute transverse myelitis. Neurology, 59:499-505.

Vitali C, Bombardieri S, Jonsson R, et al. 2002. European Study Group on Classification Criteria for Sjogren's SyndromeClassification criteria for Sjogren's syndrome: a revised version of the European criteria proposed by the American-European Consensus Group. Ann Rheum Dis, 61:554-8.

Wilson WA, Gharavi AE, Koike T, et al. 1999. International consensus statement on preliminary classification criteria for definite antiphospholipid syndrome: report of an international workshop. Arthritis Rheum, 42:1309-11. 